\title{
A reliable analytic study for higher-dimensional telegraph equation
}

\author{
Emad Az-Zo'bi \\ Department of Mathematics and Statistics, Mutah University, P. O. Box 7, Al Karak 61710-Jordan.
}

\begin{abstract}
In this study, we propose a developed semi-analytic technique, so called the generalized residual power series method, to process higher-dimensional linear and nonlinear partial differential equations. The obtained solution is expressed in a form of rapidly convergent power series with easily computable coefficients. Solution can, in turn, be termed in an exact closed form. The results indicate the reliability, efficiency, and simplicity of the proposed scheme. This is achieved by handling the $(m+1)$-dimensional hyperbolic telegraph equation.
\end{abstract}

Keywords: Generalized residual power series method, convergence analysis, exact solution, higher-dimensional partial differential equation, telegraph equation.

2010 MSC: 35C05, 35L15, 65Mxx, 65Z05.

(C)2018 All rights reserved.

\section{Introduction}

Hyperbolic partial differential equations (PDEs) are quite useful for many mathematicians because of their applications in physics and engineering [23]. These equations are represented usually by linear and nonlinear PDEs (NPDEs). Solving such equations allows physicists and engineers to draw conclusions in an efficient way, and for mathematics, such studies make the pure mathematics more meaningful. Over the last three decades, and while it is difficult to handle the nonlinear part of these equations, many techniques have been introduced for the numeric, semi-analytic, and exact analytical solutions of the NPDEs. To name few we can refer to the Adomian decomposition method (ADM) [5, 9], variational iteration method (VIM) [6, 16], homotopy analysis method (HAM) [24], homotopy perturbation method (HPM) [15], differential transform method (DTM), and its reduction (RDTM) [7, 8].

Recently, a semi-analytic technique, known as the residual power series method (RPSM), is proposed by Abu Arqub [1]. The RPSM is based on constructing power series expansion solution for nonlinear equations without transforming, linearizing, perturbation, discretization, or unnecessary assumptions with the help of residual error concepts, this method computes the coefficient of the power series by a chain of algebraic equations to get a series solution, in practice a truncated series solution. The main

Email address: eaaz2006@yahoo.com (Emad Az-Zo'bi)

doi: $10.22436 /$ jmcs.018.04.04

Received: 2018-04-12 Revised: 2018-11-11 Accepted: 2018-11-22 
advantage of this method over the other existing methods is the simplicity of direct application to nonlinear problems. The RPSM has been employed by many authors to solve ordinary differential equations of integer and fractional orders [21,32] and references therein.

The aim of the present analysis is to generalize a method to tackle linear/nonlinear higher-dimensional partial differential equations. An analytic-numeric simulation to hyperbolic telegraph equation of higherdimensional is conducted to obtain the efficiency, reliability, and accuracy of the generalized residual power series method (shortly GRPSM) [4, 10].

\section{Basic ideas of GRPSM}

To obtain a clear description of the GRPSM to handle the nonlinear problems, consider the $(m+1)$ dimensional, $\mathrm{n}^{\text {th }}$ order NPDE in general form

$$
\partial_{t}^{n} u\left(x_{i}, t\right)=F\left(t, x_{i} ; u, \partial_{t} u, \ldots, \partial_{t}^{n-1} u, \partial_{x_{i}} u, \partial_{x_{i}}^{2} u, \ldots\right),\left(x_{i}, t\right) \in R^{m} \times[0, T), i=1, \ldots, m,
$$

where $F$ is assumed to be sufficiently smooth on the indicated domain that contains the starting values. $\partial_{t}^{q} u$ represents the $q^{\text {th }}$ derivative of the analytic function $u\left(x_{1}, \ldots, x_{m}, t\right)$ with respect to independent variable $t$, and in the same way for other independent variables.

The GRPSM assumes the solution $u\left(x_{1}, \ldots, x_{m}, t\right)$, of $(2.1)$, in a form of power series

$$
u\left(x_{i}, t\right)=\sum_{j}^{\infty} \sum_{i_{1}=0}^{\infty} \ldots \sum_{i_{m}=0}^{\infty} a_{i_{1}, \ldots, i_{m}, j}\left(\prod_{k=1}^{m}\left(x_{k}-x_{k_{0}}\right)^{i_{k}}\right)\left(t-t_{0}\right)^{j} .
$$

In more compact form,

$$
u(\tilde{\mathbf{x}}, \mathrm{t})=\sum_{j=0}^{\infty} \xi_{j}(\tilde{\mathbf{x}})\left(t-t_{0}\right)^{j}
$$

where $\xi_{j}(\tilde{\mathbf{x}})=\sum_{i_{1}=0}^{\infty} \ldots \sum_{i_{m}=0}^{\infty} a_{i_{1}, \ldots, i_{m}, j}\left(\prod_{p=1}^{m}\left(x_{k}-x_{k_{0}}\right)^{i_{p}}\right)$ and $\tilde{\mathbf{x}}=\left(x_{1}, \ldots, x_{m}\right)$.

Subject to the initial conditions

$$
u\left(\tilde{\mathbf{x}}, \mathfrak{t}_{0}\right)=u_{0}(\tilde{\mathbf{x}}), \partial_{t} u\left(\tilde{\mathbf{x}}, t_{0}\right)=u_{1}(\tilde{\mathbf{x}}), \ldots, \partial_{t}^{n-1} \mathfrak{u}\left(\tilde{\mathbf{x}}, t_{0}\right)=u_{n-1}(\tilde{\mathbf{x}}),
$$

the initial approximation of $u(\tilde{\mathbf{x}}, \mathrm{t})$ will be

$$
u_{n-1}(\tilde{\mathbf{x}}, t)=\sum_{j=0}^{n-1} \frac{1}{j !} \partial_{t}^{j}\left(u\left(\tilde{x}, t_{0}\right)\right)\left(t-t_{0}\right)^{j}=\sum_{j=0}^{n-1} \frac{1}{j !} u_{j}(\tilde{\mathbf{x}})\left(t-t_{0}\right)^{j}=\sum_{j=0}^{n-1} \xi_{j}(\tilde{\mathbf{x}})\left(t-t_{0}\right)^{j} .
$$

The $k^{\text {th }}$ order approximate solution is defined by the truncated series

$$
u_{k}(\tilde{\mathbf{x}}, \mathrm{t})=u_{n-1}(\tilde{\mathbf{x}}, \mathrm{t})+\sum_{j=n}^{k} \xi_{j}(\tilde{\mathbf{x}})\left(t-t_{0}\right)^{j}, k \geqslant n,
$$

subject to

$$
\lim _{t \rightarrow t_{0}} \operatorname{Res}_{k}(\tilde{\mathbf{x}}, t)=0,
$$

where $\operatorname{Res}_{k}(\tilde{\mathbf{x}}, t)$ is the well-defined $k^{\text {th }}$ residual function defined by

$$
\operatorname{Res}_{k}(\tilde{\mathbf{x}}, t)=\partial_{t}^{k} u_{k}(\tilde{\mathbf{x}}, t)-\partial_{t}^{k-n} F\left(t, \tilde{\mathbf{x}} ; u, \partial_{t} u_{k}, \ldots, \partial_{t}^{n-1} u_{k}, \partial_{\tilde{\mathbf{x}}} u, \partial_{\tilde{\mathbf{x}}}^{2} u, \ldots\right),
$$

which represents the basic idea of the GRPSM. The exact analytic solution of the initial-value problem, (2.1) and (2.3), is given by

$$
u(\tilde{\mathbf{x}}, \mathrm{t})=\lim _{k \rightarrow \infty} \mathfrak{u}_{k}(\tilde{\mathbf{x}}, \mathrm{t}),
$$

provided that the series has exact closed form. 


\section{Convergence and error analysis}

Under considerations mentioned in the previous section, the generalized residual power series scheme approaches the exact analytic solution as more and more terms be found. This fact will be inferred through the present analytical study of convergence and error estimating for applying the GRPSM to higher-dimensional NPDEs.

Theorem 3.1. If $\mathrm{F}$ is an analytic operator on an open interval I containing $\mathrm{t}_{0}$, then the residual function $\operatorname{Res}_{\mathrm{k}}(\tilde{\mathbf{x}}, \mathrm{t})$ vanishes as $\mathrm{k}$ approaches the infinity.

Proof. It is obvious by the definition of residual function (2.7) and since F is assumed to be analytic.

Lemma 3.2. Suppose that $\mathrm{u}(\tilde{\mathbf{x}}, \mathrm{t})=\sum_{j=0}^{\infty} \xi_{\mathbf{j}}(\tilde{\mathbf{x}})\left(\mathrm{t}-\mathrm{t}_{0}\right)^{\mathrm{j}}$, then

$$
\left[\partial_{\mathrm{t}}^{\mathrm{q}} \mathrm{u}(\tilde{\mathbf{x}}, \mathrm{t})\right]_{\mathrm{t}=\mathrm{t}_{0}}=\mathrm{q} ! \xi_{\mathrm{q}}(\tilde{\mathbf{x}}), \mathrm{q} \in \mathrm{N} .
$$

Proof. The $\mathrm{q}^{\text {th }}$ derivative of $u(x, t)$ with respect to $t$ is continuous at $t=t_{0}$. Therefore

$$
\begin{aligned}
& {\left[\partial_{t}^{q} u(\tilde{x}, t)\right]_{t=t_{0}}=\lim _{t \rightarrow t_{0}} \partial_{t}^{q} u(x, t)} \\
& =\lim _{\mathrm{t} \rightarrow \mathrm{t}_{0}} \partial_{\mathrm{t}}^{\mathrm{q}}\left(\sum_{i=0}^{\infty} \xi_{j}(\tilde{\mathbf{x}})\left(\mathrm{t}-\mathrm{t}_{0}\right)^{j}\right) \\
& =\lim _{\mathrm{t} \rightarrow \mathrm{t}_{0}}\left(\sum_{i=0}^{\infty} \xi_{j}(\tilde{\mathbf{x}}) \partial_{\mathrm{t}}^{\mathrm{q}}\left(\mathrm{t}-\mathrm{t}_{0}\right)^{j}\right) \\
& =\lim _{t \rightarrow t_{0}}\left(\sum_{i=0}^{\infty} \frac{(q+j) !}{j !} \xi_{q+j}(\tilde{\mathbf{x}})\left(t-t_{0}\right)^{j}\right) \\
& =\sum_{i=0}^{\infty}\left(\frac{(q+j) !}{j !} \xi_{q+j}(\tilde{\mathbf{x}}) \lim _{\mathrm{t} \rightarrow \mathrm{t}_{0}}\left(\mathrm{t}-\mathrm{t}_{0}\right)^{j}\right)=\mathrm{q} ! \xi_{\mathrm{q}}(\tilde{\mathbf{x}}) .
\end{aligned}
$$

As a result of Theorem 3.1,

$$
u^{(0, k)}\left(x, t_{0}\right)=-\lim _{t \rightarrow t_{0}} \partial_{t}^{k-n}\left(L_{x} u_{k}+R u_{k}+N u_{k}-H(x, t)\right),
$$

which completes the proof.

Theorem 3.3. The approximate truncated series solution $\mathfrak{u}_{\mathrm{k}}(\tilde{\mathbf{x}}, \mathrm{t})$ defined in (2.5) and obtained by applying the GRPSM for solving the (2.1) and (2.3) is the $\mathrm{k}^{\text {th }}$ Taylor polynomial of $\mathrm{u}(\tilde{\mathbf{x}}, \mathrm{t})$ about $\mathrm{t}=\mathrm{t}_{0}$. In general, as $\mathrm{k} \rightarrow \infty$, the series solution in (2.8) concise the Taylor series expansion of $\mathrm{u}(\tilde{\mathbf{x}}, \mathrm{t})$ centered at $\mathrm{t}=\mathrm{t}_{0}$.

Proof. For $k<n$, it is clear from the initial approximation of $u(\tilde{\mathbf{x}}, t)$ in (2.4). For $k \geqslant n$, it suffices to prove that

$$
\left[\partial_{t}^{k} u(\tilde{x}, t)\right]_{t=t_{0}}=\lim _{t \rightarrow t_{0}} \partial_{t}^{k-n} F\left(t, \tilde{x} ; u, \partial_{t} u_{k}, \ldots, \partial_{t}^{n-1} u_{k}, \partial_{\tilde{x}} u, \partial_{\tilde{x}}^{2} u, \ldots\right) .
$$

Applying (2.6) to the $k^{\text {th }}$ order approximate solution given in (2.5), and using the result of Theorem 3.1, we get

$$
\begin{aligned}
& 0=\lim _{k \rightarrow \infty} \operatorname{Res}_{k}(\tilde{\mathbf{x}}, t)=\lim _{t \rightarrow t_{0}}\left(\partial_{t}^{k} u_{k}(\tilde{\mathbf{x}}, t)-\partial_{t}^{k-n} F\left(t, \tilde{\mathbf{x}} ; \mathfrak{u}, \partial_{t} u_{k}, \ldots, \partial_{t}^{n-1} \mathfrak{u}_{k}, \partial_{\tilde{\mathbf{x}}} u, \partial_{\tilde{\mathbf{x}}}^{2} u, \ldots\right)\right) \\
& =\lim _{t \rightarrow t_{0}} \partial_{t}^{k} u_{k}(\tilde{x}, t)-\lim _{t \rightarrow t_{0}} \partial_{t}^{k-n} F\left(t, \tilde{x} ; u, \partial_{t} u_{k}, \ldots, \partial_{t}^{n-1} u_{k}, \partial_{\tilde{x}} u, \partial_{\tilde{\mathbf{x}}}^{2} u, \ldots\right) .
\end{aligned}
$$


As a result of Lemma 3.2,

$$
0=q ! \xi_{q}(\tilde{\mathbf{x}})-\lim _{t \rightarrow t_{0}} \partial_{t}^{k-n} F\left(t, \tilde{x} ; u, \partial_{t} u_{k}, \ldots, \partial_{t}^{n-1} u_{k}, \partial_{\tilde{x}} u, \partial_{\tilde{x}}^{2} u, \ldots\right)
$$

which completes the proof.

Corollary 3.4. Suppose that the truncated series $\mathrm{u}_{\mathrm{k}}(\mathrm{x}, \mathrm{t})(2.5)$ is used as an approximation to the solution $\mathrm{u}(\mathrm{x}, \mathrm{t})$ of problem (2.1) and (2.3) on a strip

$$
S=\left\{(\tilde{\mathbf{x}}, \mathrm{t}): \tilde{\mathbf{x}} \in R^{\mathrm{m}},\left|\mathrm{t}-\mathrm{t}_{0}\right|<\rho\right\},
$$

then numbers $\eta(t)$, satisfy $\left|\eta(t)-t_{0}\right| \leqslant \rho$, and $\mu_{k}>0$ exists with

$$
\left|u(\tilde{\mathbf{x}}, t)-u_{k}(\tilde{\mathbf{x}}, t)\right| \leqslant \frac{\mu_{k}}{(k+1) !} \rho^{k+1} .
$$

Proof. Theorem 3.1 implies that

$$
u(\tilde{\mathbf{x}}, t)-u_{k}(\tilde{\mathbf{x}}, t)=\sum_{j=k+1}^{\infty} \frac{1}{j !} \partial_{t}^{j}(u(\tilde{x}, t))\left(t-t_{0}\right)^{j} .
$$

Following the proof of Taylor's Theorem [12], a number $\eta(t) \in\left(t_{0}-\rho, t_{0}+\rho\right)$ exists with

$$
u(\tilde{\mathbf{x}}, \mathrm{t})-\mathfrak{u}_{\mathrm{k}}(\tilde{\mathbf{x}}, \mathrm{t})=\frac{\partial_{\mathrm{t}}^{\mathrm{k}+1} \mathfrak{u}(\tilde{\mathbf{x}}, \eta(t))}{(k+1) !}\left(\mathrm{t}-\mathrm{t}_{0}\right)^{\mathrm{k}+1}
$$

Since the $(k+1)$ st-derivative of the analytic function $u(x, t)$ with respect to $t$ is bounded on $S$, a number $\mu_{k}$ also exists with $\left|\partial_{t}^{k+1} u(\tilde{\mathbf{x}}, t)\right| \leqslant \mu_{k}$ for all $t \in\left[t_{0}-\rho, t_{0}+\rho\right]$. Hence we get the result.

Corollary 3.5. The GRPSM results the exact analytic solution $\mathrm{u}(\tilde{\mathbf{x}}, \mathrm{t})$ if it is a polynomial of $\mathrm{t}$.

\section{Generalized hyperbolic telegraph equation}

The telegraph equation was first introduced by Kirchhoff in 1857. But it was first examined by Poincare in 1893. The telegraph equation has both wave motion and diffusion properties. For example, in fluid dynamics, it shows the random motion of the particle, traveling of electromagnetic waves in super conducting media and propagation of pressure waves occurring in pulsatile blood flow in arteries [28]. It also describes wave propagation [33], random walk theory [11], and signal analysis [20].

The m-space-dimensional hyperbolic second-order telegraph equation is of the form

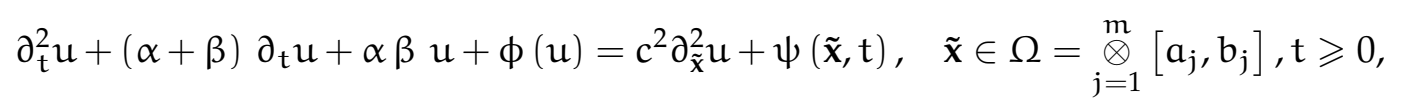

where $u(\tilde{\mathbf{x}}, t)$ denotes either the voltage or current through the $m$-dimensional conductor at position $\tilde{\mathbf{x}}=\left(x_{1}, \ldots, x_{m}\right)$ at the time $t, \alpha=G / C, \beta=R / I, c^{2}=1 / I C, G$ is the conductance of a resistor, $R$ is the resistance of the resistor, $I$ is the inductance of the coil and $C$ is the capacitance of the capacitor, $\psi(\tilde{\mathbf{x}}, t)$ is the inhomogeneous term, which is assumed to be analytic in time $t$, and $\phi(u)$ is the continuous nonlinear resorting term. For $\alpha>0$ and $\beta=0$, (4.1) models the damped wave equation in $m$ dimensions.

Recently, many iterative methods were applied to handle various forms of telegraph equation. See $[2,3,13,14,17-19,22,25-27,29-31]$ and references therein. To illustrate the methodology of our technique discussed in Sections 2, consider (4.1) subject to the initial conditions

$$
u\left(\tilde{\mathbf{x}}, \mathrm{t}_{0}\right)=\mathrm{u}_{0}(\tilde{\mathbf{x}}), \quad \partial_{\mathrm{t}} \mathrm{u}\left(\tilde{\mathbf{x}}, \mathrm{t}_{0}\right)=\mathrm{u}_{1}(\tilde{\mathbf{x}}), \quad \tilde{\mathbf{x}} \in \Omega, \mathrm{t}_{0} \geqslant 0 .
$$


The initial approximate solution is given in (2.4) for $n=2$, that is

$$
\mathbf{u}_{1}(\tilde{\mathbf{x}}, \mathrm{t})=\xi_{0}(\tilde{\mathbf{x}})+\xi_{1}(\tilde{\mathbf{x}})\left(t-t_{0}\right),
$$

where $\xi_{0}(\tilde{\mathbf{x}})=\mathfrak{u}_{0}(\tilde{\mathbf{x}})$ and $\xi_{1}(\tilde{\mathbf{x}})=\mathfrak{u}_{1}(\tilde{\mathbf{x}})$. The $k^{\text {th }}$ order approximate solution is defined by

$$
u_{k}(\tilde{\mathbf{x}}, t)=u_{1}(\tilde{\mathbf{x}}, t)+\sum_{j=2}^{k} \xi_{j}(\tilde{\mathbf{x}})\left(t-t_{0}\right)^{j}, k \geqslant 2 .
$$

For each k,

$$
\operatorname{Res}_{k}(\tilde{\mathbf{x}}, t)=\partial_{t}^{k} u_{k}(\tilde{\mathbf{x}}, t)+(\alpha+\beta) \partial_{t}^{k-1} \mathfrak{u}_{k}(\tilde{\mathbf{x}}, t)+\partial_{t}^{k-2}\left[\alpha \beta u_{k}+\phi\left(u_{k}\right)-c^{2} \partial_{\tilde{\mathbf{x}}}^{2} \mathfrak{u}_{k}-\psi(\tilde{\mathbf{x}}, t)\right]
$$

is the associated residual function which satisfies $\lim _{t \rightarrow t_{0}} \operatorname{Res}_{k}(\tilde{\mathbf{x}}, t)=0$. Following the mentioned procedure, and by solving the following equation

$$
2 \xi_{2}(\tilde{\mathbf{x}})+(\alpha+\beta) \xi_{1}(\tilde{\mathbf{x}})+\alpha \beta \xi_{0}(\tilde{\mathbf{x}})+\phi\left(\xi_{0}(\tilde{\mathbf{x}})\right)-c^{2} \partial_{\tilde{\mathbf{x}}}^{2} \xi_{0}(\tilde{\mathbf{x}})-\psi\left(\tilde{\mathbf{x}}, \mathrm{t}_{0}\right)=0,
$$

in term of $\xi_{2}(\tilde{\mathbf{x}})$, and by continuity of nonlinear operator $\phi$, the first term of truncated series solution $(k=2)$ in $(4.2)$ would be determined to be

$$
\xi_{2}(\tilde{\mathbf{x}})=-\frac{1}{2}\left[(\alpha+\beta) \xi_{1}(\tilde{\mathbf{x}})+\alpha \beta \xi_{0}(\tilde{\mathbf{x}})+\phi\left(\xi_{0}(\tilde{\mathbf{x}})\right)-c^{2} \partial_{\tilde{\mathbf{x}}}^{2} \xi_{0}(\tilde{\mathbf{x}})-\psi\left(\tilde{\mathbf{x}}, \mathrm{t}_{0}\right)\right] .
$$

Repeating the previous step for $k=3$, the next unknown coefficient $\xi_{3}(\tilde{\mathbf{x}})$ of approximate series solution is calculated and got the form

$$
\xi_{3}(\tilde{\mathbf{x}})=-\frac{1}{6}\left[2(\alpha+\beta) \xi_{2}(\tilde{\mathbf{x}})+\alpha \beta \xi_{1}(\tilde{\mathbf{x}})+\xi_{1}(\tilde{\mathbf{x}}) \partial_{\xi_{0}} \phi\left(\xi_{0}(\tilde{\mathbf{x}})\right)-c^{2} \partial_{\tilde{\mathbf{x}}}^{2} \xi_{0}(\tilde{\mathbf{x}})-\psi\left(\tilde{\mathbf{x}}, \mathrm{t}_{0}\right)\right] .
$$

Continuing as before, more and more series solution coefficients can be obtained easily. The local truncation error is $\mathrm{O}(\mathrm{k}+1)$.

\section{Numerical illustration}

To validate the efficiency and reliability of the aforesaid technique, a higher dimensional test problem with quadratic nonlinear restoring is presented.

$$
\partial_{t}^{2} u(\tilde{\mathbf{x}}, t)+2 \partial_{t} u(\tilde{\mathbf{x}}, t)+u(\tilde{\mathbf{x}}, t)^{2}=\sum_{j=1}^{m} \partial_{x_{j}}^{2} u(\tilde{\mathbf{x}}, t)+e^{-4 t+2 \sum_{j=1}^{m} x_{j}}-m e^{-2 t+\sum_{j=1}^{m} x_{j}},
$$

subject to the initial data

$$
u(x, 0)=e^{\sum_{j=1}^{m} x_{j}}, \quad \partial_{t} u(x, 0)=-2 e^{\sum_{j=1}^{m} x_{j}} .
$$

Following the analysis operated in previous section for solving telegraph equations, and with the aid of Mathematica software, we list the first few coefficients of series solution (2.2):

$$
\xi_{0}(x)=e^{\sum_{j=1}^{m} x_{j}}, \quad \xi_{1}(x)=-2 e^{\sum_{j=1}^{m} x_{j}}, \quad \xi_{2}(x)=2 e^{\sum_{j=1}^{m} x_{j}}, \quad \xi_{3}(x)=-\frac{4}{3} e^{\sum_{j=1}^{m} x_{j}}, \quad \xi_{4}(x)=\frac{2}{3} e^{\sum_{j=1}^{m} x_{j}}, \quad \ldots
$$

The general formula for the $k^{\text {th }}$ term is $\xi_{k}(x)=\frac{(-2)^{k}}{k !} e^{\sum^{m} x^{1}}$. As $k \rightarrow \infty$, the solution takes the form

$$
u(\tilde{\mathbf{x}}, \mathrm{t})=e^{\sum_{j=1}^{m} x_{j}} \sum_{k=1}^{\infty} \frac{(-2)^{k}}{k !} t^{k}=e^{-2 t+\sum_{j=1}^{m} x_{j}} .
$$

The series solution leads to the exact solution obtained by Taylor's expansion. 


\section{Conclusions}

In this paper, the generalized residual power series method is devised to tackle multi-dimensional partial differential equations. The solution using new approach is given in series form without any need for perturbation techniques, special transformations, linearization or discretization. The method overcomes the complexity of computing integrals in some recent techniques and the large size of calculations in the others. The convergence proof and error analysis of the aforesaid technique are discussed deeply. The $\mathrm{m}$-space-dimensional hyperbolic second-order telegraph equation is considered for finding exact analytic solution via generalized residual power series method. It is observed that the presented methodology is very powerful and efficient with high accuracy.

\section{Acknowledgment}

The author would like to express his sincere grateful to the reviewers for their valuable comments.

\section{References}

[1] O. Abu Arqub, Z. Abo-Hammour, R. Al-Badarneh, S. Momani, A reliable analytical method for solving higher-order initial value problems, Discrete Dyn. Nat. Soc., 2013 (2013), 12 pages. 1

[2] J. Ahmad, G. Mohiuddin, Analytical exact solution of telegraph equation using HPM, BIBECHANA, 14 (2017), 30-36. 4

[3] H. Al-badrani, S. Saleh, H. O. Bakodah, M. Al-Mazmumy, Numerical solution for nonlinear telegraph equation by modified Adomian decomposition method, Nonlinear Anal. Diff. Equ., 4 (2016), 243-257. 4

[4] E. A. Az-Zo'bi, Exact analytic solutions for nonlinear diffusion equations via generalized residual power series method, Int. J. Math. Comput. Sci., 14 (2019), 69-78. 1

[5] E. A. Az-Zo'bi, Construction of Solutions for Mixed Hyperbolic Elliptic Riemann Initial Value System of Conservation Laws, Appl. Math. Model., 37 (2013), 6018-6024. 1

[6] E. A. Az-Zo'bi, On the convergence of variational iteration method for solving systems of conservation laws, Trends Appl. Sci. Res., 10 (2015), 157-165. 1

[7] E. A. Az-Zo'bi, On the reduced differential transform method and its application to the generalized Burgers-Huxley equation, Appl. Math. Sci., 8 (2014), 8823-8831. 1

[8] E. A. Az-Zo'bi, K. Al Dawoud, M. Marashdeh, Numeric-analytic solutions of mixed-type systems of balance laws, Appl. Math. Comput., 265 (2015), 133-143. 1

[9] E. A. Az-Zo'bi, K. Al-Khaled, A new convergence proof of the Adomian decomposition method for a mixed hyperbolic elliptic system of conservation laws, Appl. Math. Comput., 217 (2010), 4248-4256. 1

[10] E. A. Az-Zo'bi, A. Yildirim, W. A. AlZoubi, The residual power series method for the one-dimensional unsteady flow of a van der Waals gas, Phys. A, 517 (2019), 188-196. 1

[11] J. Banasiak, J. R. Mika, Singularly perturbed telegraph equations with applications in the random walk theory, J. Appl. Math. Stochastic Anal., 11 (1998), 9-28. 4

[12] R. G. Bartle, D. R. Sherbert, Introduction to Real Analysis, John Wiley \& Sons, Hoboken, (2011). 3

[13] S. Das, P. K. Gupta, Homotopy analysis method for solving fractional hyperbolic partial differential equations, Int. J. Comput. Math., 88 (2011), 578-588. 4

[14] A. Z. Fino, H. Ibrahim, Analytical solution for a generalized space-time fractional telegraph equation, Math. Methods Appl. Sci., 36 (2013), 1813-1824. 4

[15] J. H. He, A coupling method of homotopy technique and perturbation technique for nonlinear problems, Int. J. Non-Linear Mech., 35 (2000), 37-43. 1

[16] J. H. He, Variational iteration approach to nonlinear problems and its applications, Mech. Appl., 20 (1998), 30-31. 1

[17] E. Hesameddini, E. Asadolahifard, The sinc-collocation method for solving the telegraph equation, J. Comput. Eng. Infor., 1 (2013), 13-17. 4

[18] T. S. Jang, A new solution procedure for the nonlinear telegraph equation, Commun. Nonlinear Sci. Numer. Simul., 29 (2015), 307-326.

[19] M. Javidi, N. Nyamoradi, Numerical solution of telegraph equation by using LT inversion technique, Int. J. Adv. Math. Sci., 1 (2013), 64-77. 4

[20] P. M. Jordan, A. Puri, Digital signal propagation in dispersive media, J. Appl. Phys., 85 (1999), 1273-1282. 4

[21] A. Kumar, S. Kumar, Residual power series method for fractional Burger types equations, Nonlinear Eng., 5 (2016), 235-244. 1

[22] M. Lakestani, B. N. Saray, Numerical solution of telegraph equation using interpolating scaling functions, Comput. Math. Appl., 60 (2010), 1964-1972. 4

[23] P. D. Lax, Hyperbolic partial differential equations, American Mathematical Society, Providence (2006). 1 
[24] S.-J. Liao, An explicit, totally analytic approximate solution for Blasius' viscous flow problems, Internat. J. Non-Linear Mech., 34 (1999), 759-778. 1

[25] R. C. Mittal, R. Bhatia, A numerical study of two dimensional hyperbolic telegraph equation by modified B-spline differential quadrature method, Appl. Math. Comput., 244 (2014), 976-997. 4

[26] R. Rajaraman, Analytical solutions for the different forms of telegraph equations by homotopy analysis method, Global J. Sci. Frontier Research, 12 (2012), 7-12.

[27] M. Rawashdeh, N. A. Obeidat, On finding exact and approximate solutions to some PDEs using the reduced differential transform method, Appl. Math. Inf. Sci., 8 (2014), 2171-2176. 4

[28] R. J. Schwarz, B. Friedland, Linear Systems, McGraw-Hill, New York, (1965). 4

[29] B. Soltanalizadeh, Differential transformation method for solving one-space-dimensional telegraph equation, Comput. Appl. Math., 30 (2011), 639-653. 4

[30] V. K. Srivastava, M. K. Awasthi, R. K. Chaurasia, Reduced differential transform method to solve two and three dimensional second order hyperbolic telegraph equations, J. King Saud University-Eng. Sci., 29 (2017), 166-171.

[31] V. K. Srivastava, M. K. Awasthi, R. K. Chaurasia, M. Tamsir, The telegraph equation and its solution by reduced differential transform method, Model. Simul. Eng., 2013 (2013), 6 pages. 4

[32] M. I. Syam,Analytical solution of the fractional initial Emden-Fowler equation using the fractional residual power series method, Int. J. Appl. Comput. Math., 4 (2018), 8 pages. 1

[33] V. H. Weston, S. He, Wave splitting of the telegraph equation in R3 and its application to inverse scattering, Inverse Prob., 9 (1993), 789-812. 4 